\title{
Exchange Coupling Effects in Naturally Oxidised Ultrathin Iron Film
}

\author{
H. DAWCZAK-DęBicki ${ }^{a, c}$, A. Marczyńska ${ }^{a}$, A. RogowskA $^{a, b}$, M. WACHOWiak $^{a, b}$,

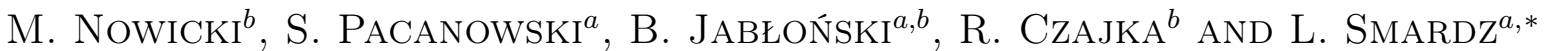 \\ ${ }^{a}$ Institute of Molecular Physics, Polish Academy of Sciences, \\ M. Smoluchowskiego 17, 60-179 Poznań, Poland \\ ${ }^{b}$ Faculty of Technical Physics, Poznań University of Technology, Piotrowo 3, 60-965 Poznań, Poland \\ ${ }^{c}$ Faculty of Physics, Adam Mickiewicz University, Umultowska 85, 61-614 Poznań, Poland
}

Oxidation process of Fe films under atmospheric conditions is depth limited such that an oxide covering layer with a well-defined thickness is formed by which the underlying metal is prevented from further oxidation. Iron thin film with an initial thickness $d_{i}=4 \mathrm{~nm}$ was deposited onto $1.6 \mathrm{~nm}-\mathrm{V}(110)$ buffer layer using UHV magnetron sputtering. The planar growth of $\mathrm{Fe}$ oxides was revealed by atomic force microscopy. X-ray photoelectron spectroscopy studies performed after 250 days of oxidation revealed formation of a hematite $\left(\alpha-\mathrm{Fe}_{2} \mathrm{O}_{3}\right)$ ultrathin film on the metallic rest of iron. Furthermore, low temperature magnetic measurements of the oxidised Fe ultrathin film revealed an exchange anisotropy which is imposed to the metallic rest. As a result, we have observed at low temperatures a shift and broadening of the hysteresis loops due to the exchange interaction at the metal-oxide interface.

DOI: 10.12693/APhysPolA.133.601

PACS/topics: $75.70 .-\mathrm{i} ; 75.70 . \mathrm{Kw}$

\section{Introduction}

As is well known, all metallic thin films oxidize under atmospheric conditions. Normally this process is depth limited such that an oxide covering layer with a well-defined thickness is formed by which the underlying metal is prevented from further oxidation. In this way one can obtain a self-stabilized metal/oxide bilayer structure [1-3].

Binary iron oxides range from reasonable conductors (magnetite $-\mathrm{Fe}_{3} \mathrm{O}_{4}$ ) to insulators and from ferrimagnets to antiferromagnets [4-5]. Hematite $\left(\alpha-\mathrm{Fe}_{2} \mathrm{O}_{3}\right)$, the most stable phase in ambient conditions, is a canted antiferromagnet (weak ferromagnet) with the corundum structure.

The iron thin film investigated here, after exposition to ambient conditions actually consists of bilayer system with two magnetically active materials, namely nonoxidised metallic iron covered by natural oxide layer. In Fig. 1 we show a schematic description of a transformation process of the "as prepared" Fe thin film (left panel) after extraction from ultra high vacuum (UHV) to ambient conditions (right panel). The oxidized surface layer is predominantly consisted of $\alpha-\mathrm{Fe}_{2} \mathrm{O}_{3}$. Such a layer practically gives no magnetic signal at room temperature compared to that measured for nonoxidised metallic Fe layer. Metallic bcc iron, on the other hand, is a well-known ferromagnet with a magnetic moment per Fe atom of about

*corresponding author; e-mail: smardz@ifmpan.poznan.pl
$2.2 \mu_{\mathrm{B}}$. Formation of oxide is at the expense of the metallic Fe ferromagnet and therefore associated with a loss of the observed total saturation magnetic moment. Using a sensitive vibrating sample magnetometer (VSM) we have measured the hysteresis curves of such an iron film on an absolute scale and could evaluate a quantitative measure for the amount of ferromagnetic metal being transformed into approximately nonmagnetic oxide at room temperature. Preliminary results on oxidation kinetics were already published in Ref. [6].

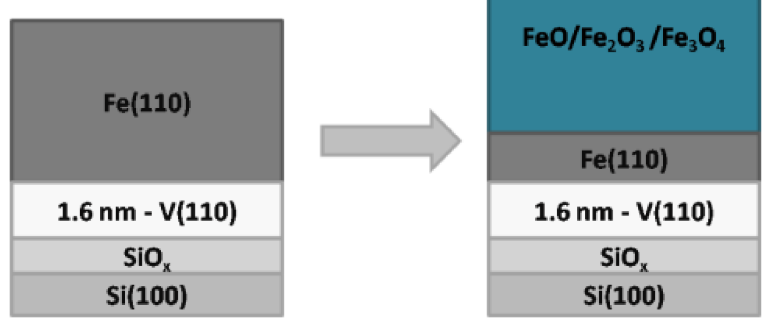

Fig. 1. Schematic description of "as prepared" (left panel) and oxidised under ambient conditions (right panel) iron thin film.

With decreasing iron thickness one further interesting phenomenon could be observed at low temperatures, brought about by an increasing importance of the interface between metal and oxide. As has been discovered long before [7-8], the magnetic switching properties of the ferromagnetic Fe metal are strongly changed by the action of the adjacent oxide which imposes a new type 
of anisotropy onto the Fe metal, the so-called exchangebiasing (EB) or unidirectional anisotropy [1-3, 7-10]. In this paper we show results on exchange coupling effects in naturally oxidised ultra thin Fe film (see Fig. 1, right panel).

\section{Experimental procedure}

The Fe thin films with thicknesses of about $4 \mathrm{~nm}$ were prepared at room temperature on $1.6 \mathrm{~nm} \mathrm{~V}$ buffer layer using UHV $\left(5 \times 10^{-10}\right.$ mbar $)$ magnetron sputtering [11-14]. As a substrate we have used $\mathrm{Si}(100)$ wafer with an oxidised surface to prevent a silicide formation [15-17]. The Fe-layer was deposited using a DC source. For preparation of $\mathrm{V}$ buffer layer a RF source was used.

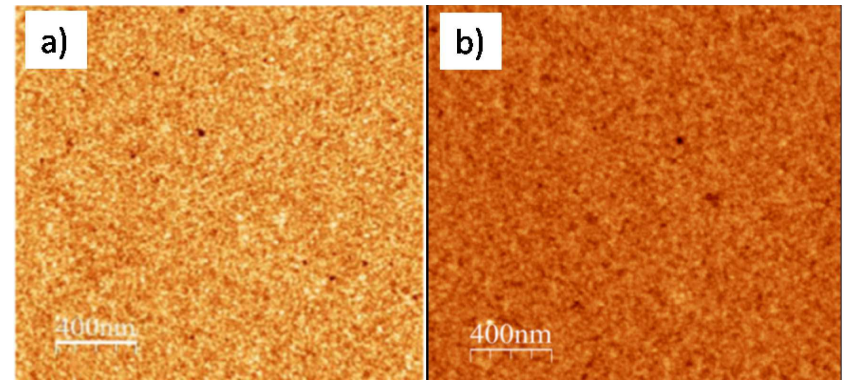

Fig. 2. AFM images measured for the Fe film with $d_{i}=$ $4 \mathrm{~nm}$ after 5 (a) and 144 days (b) of oxidation under ambient conditions.

The chemical composition and the cleanness of all layers were checked in-situ, immediately after deposition, transferring the samples to an UHV $\left(4 \times 10^{-11}\right.$ mbar $)$ analysis chamber equipped with $\mathrm{X}$-ray Photoelectron Spectroscopy (XPS). The XPS spectra were measured at room temperature using a SPECS EA 10 PLUS energy spectrometer with $\mathrm{Al}-\mathrm{K}_{\alpha}$ radiation of $1486.6 \mathrm{eV}$. All emission spectra were measured immediately after the sample transfer in vacuum of $8 \times 10^{-11}$ mbar. Details of the XPS measurements can be found in Ref. [18-20]. The morphology and roughness of the oxide layers were studied ex-situ by atomic force microscopy (AFM). The magnetic measurements of the oxidised sample were carried out using a sensitive VSM in the temperature range $10-300 \mathrm{~K}$. The hysteresis loops were measured in an inplane magnetic field up to $5 \mathrm{~T}$. Before the measurements the sample was cooled down from $300 \mathrm{~K}$ to $10 \mathrm{~K}$ in a positive magnetic field of about $2 \mathrm{~T}$.

\section{Results and discussion}

After extraction from UHV preparation chamber and exposition to ambient conditions (air at room temperature) the iron thin film with an initial thickness $d_{i}$ equal to $4 \mathrm{~nm}$ starts to oxidise. Formation of oxide is at the expense of the metallic Fe ferromagnet (see Fig. 1) and therefore associated with a loss of the observed saturation magnetic moment. Using a sensitive VSM we have measured the hysteresis curves of such iron films on an absolute scale and could evaluate a quantitative measure for the amount of ferromagnetic metal being transformed into nonmagnetic oxide at room temperature [6].

In Fig. 2 we show AFM images obtained for the $4 \mathrm{~nm}$ Fe thin film after 5 (a) and 144 days (b) of oxidation under ambient conditions at room temperature. The images show actual morphology of the oxidised Fe top layer. The roughness parameter $\left(R_{a}\right)$ measured for $2 \mu \mathrm{m} \times 2 \mu \mathrm{m}$ area was about $0.20 \mathrm{~nm}$ and $0.22 \mathrm{~nm}$ for the sample after 5 and 144 days of oxidation, respectively. The relatively low values of the roughness parameters revealed planar growth of an oxide layer on the metallic rest of the iron film. Fig. 3a shows XPS spectrum of freshly deposited $4 \mathrm{~nm}-\mathrm{Fe}(110)$ thin film on $1.6 \mathrm{~nm}-\mathrm{V}(110)$ buffer layer. Details concerning growth and structural characterisation of the $\mathrm{Fe} / \mathrm{V}$ bilayers were published elsewhere [21-22]. XPS results showed no signal from potential contamination atoms like $\mathrm{O}: 1 \mathrm{~s}$ and $\mathrm{C}: 1 \mathrm{~s}$.
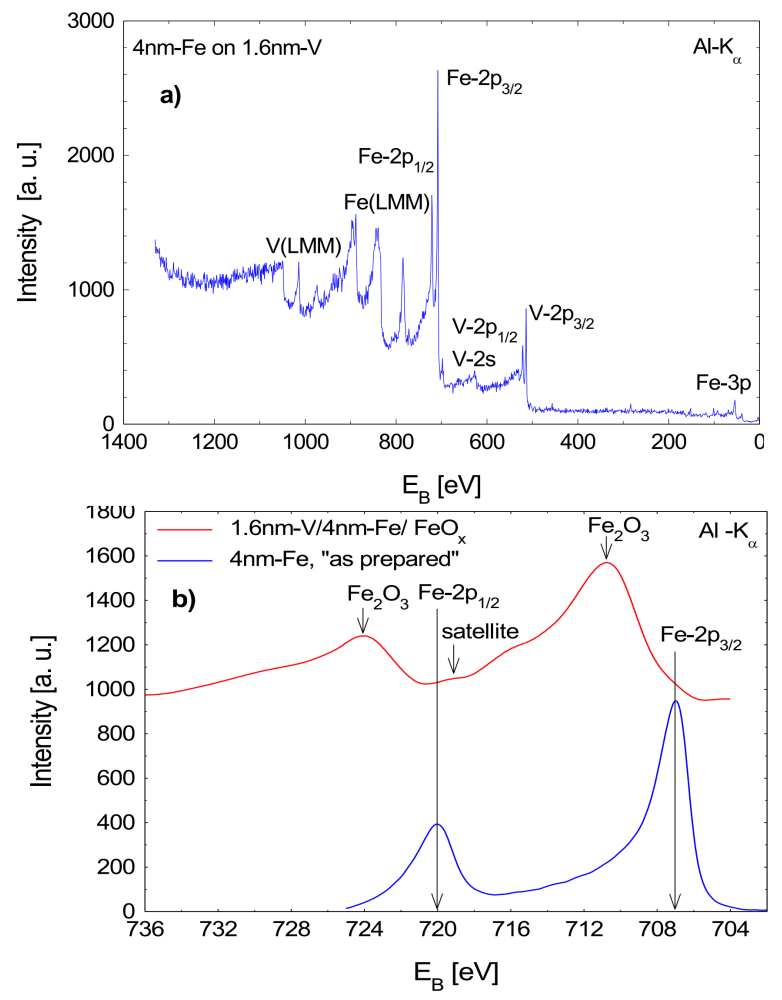

Fig. 3. XPS (Al-K) spectrum (a) of "as prepared" Fe thin film with an initial Fe thickness $d_{i}=4 \mathrm{~nm}$, measured in-situ immediately after preparation. XPS spectra near Fe- $2 p$ peaks (b) for the "as prepared" and oxidised sample after 250 days of oxidation under ambient conditions.

X-ray photoelectron spectroscopy is an universal surface analysis technique that can be used for compositional and chemical states analysis. Since X-rays are used for the incident beam, the XPS technique causes 
very little charging of samples and thus it is useful for both electrically conductive and non-conductive materials. The information depth depends on the electron kinetic energy and emission angle [23]. For the electron energies that are normally used in XPS, the information depth ranges from 1 to 3 nanometres for the emission angles normal to the surface [23]. The peak positions of $\mathrm{Fe}-2 p_{1 / 2}$ and $\mathrm{Fe}-2 p_{3 / 2}$ depend on the ionic states of $\mathrm{Fe}$. The positions of the satellite peaks for the Fe- $2 p_{1 / 2}$ and Fe- $2 p_{3 / 2}$ peaks are also very sensitive to the oxidation states [23] and these peaks have been used for qualitatively determining the ionic states of iron. Therefore, the XPS measurements were also curried out near the Fe- $2 p$ peaks for the "as prepared" and oxidised sample. The corresponding XPS peaks of the Fe- $2 p_{3 / 2}$ and Fe- $2 p_{1 / 2}$ are shown in Fig. 3b. In the case of the "as prepared" thin film the Fe- $2 p_{3 / 2}$ peak is narrower and stronger than Fe$2 p_{1 / 2}$ and the area of $\mathrm{Fe}-2 p_{3 / 2}$ peak is greater than that of Fe- $2 p_{1 / 2}$. The shifted positions of the Fe- $2 p$ peaks for the oxidised sample and weak satellite peak (see Fig. 3b) revealed formation of the $\mathrm{Fe}_{2} \mathrm{O}_{3}$ (hematite) ultra-thin film after 250 days of oxidation.
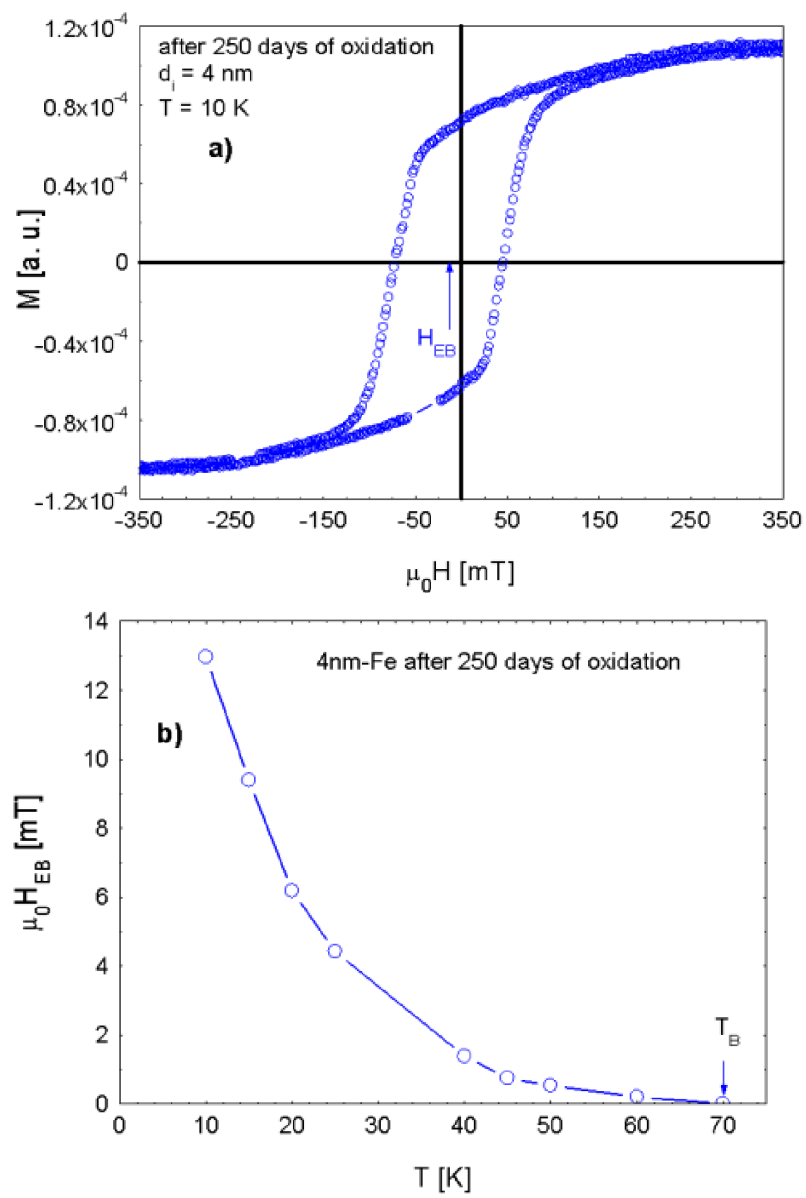

Fig. 4. Hysteresis loop of the Fe film with $d_{i}=4 \mathrm{~nm}$ measured at $10 \mathrm{~K}$ after 250 days of oxidation (a) and temperature dependence of the exchange bias field (shift of the hysteresis loop) (b).
In Fig. 4a we show hysteresis loop for the oxidised sample measured at $10 \mathrm{~K}$. The hysteresis loop is displaced from the origin and broadened compared to that measured for the Fe layer of the same thickness but protected against oxidation by $5 \mathrm{~nm}-\mathrm{Pd}$ layer. The value of this displacement $\left(H_{E B}\right)$ defines directly the exchange biasing (unidirectional anisotropy) field. The hysteresis loop experiences a field offset that opposes the field direction in which the system has been cooled through the Neél temperature of the oxide. In our case, the bilayer was cooled from $300 \mathrm{~K}$ to $10 \mathrm{~K}$ in a positive magnetic field of about $2 \mathrm{~T}$. The temperature dependence of the EB field is shown in Fig. 4b. The EB field decreases monotonically up to a blocking temperature $T_{b} \approx 70 \mathrm{~K}$. Basing on the XPS measurements of the "as prepared" and oxidised ultrathin iron film we can also rule out chemical impurities like carbonates at the interface as a factor reducing the EB field. Therefore, the estimated relatively low $T_{b}$ value could be associated with a low ordering temperature of the oxide. Based on the magnetic hysteresis measurements we have estimated the thickness of the oxidized and non-oxidized part of the film as $\sim 4$ and $\sim 1.5 \mathrm{~nm}$, respectively. Details will be published in the separate paper.

In conclusion, XPS studies of the $4 \mathrm{~nm}-$ Fe thin film after 250 days of natural oxidation revealed formation of the $\alpha-\mathrm{Fe}_{2} \mathrm{O}_{3}$ (hematite) ultrathin film on the metallic rest. Due to exchange interaction at the oxide - metal interface we have observed exchange coupling effects up to $T_{b} \approx 70 \mathrm{~K}$.

\section{References}

[1] L. Smardz, U. Köbler, W. Zinn, J. Appl. Phys. 71, 5199 (1992)

[2] L. Smardz, U. Köbler, W. Zinn, Vacuum 42, 283 (1991).

[3] J.D. Baran, H. Grönbeck, A. Hellman, Phys. Rev. Lett. 112, 146103 (2014).

[4] R. Cornell, U. Schwertmann, The Iron Oxides, Wiley \& Sons, New York 1997.

[5] M. Monti, B. Santos, A. Mascaraque, O. Rodriguez de la Fuente, M.A. Niño, T.O. Menteţ, A. Locatelli, K.F. McCarty, J. F. Marco and J. de la Figuera, J. Phys. Chem. C 116, 11539 (2012).

[6] A. Marczyńska, J. Skoryna, M. Lewandowski, L. Smardz, Acta Phys. Pol. A 127, 549 (2015).

[7] W.H. Meiklejohn, J. Appl. Phys. 33, 1328 (1962).

[8] A. P. Malozemoff, J. Appl. Phys. 63, 3874 (1988).

[9] L. Smardz, phys. stat. sol. (a) 181, R7 (2000).

[10] L. Smardz, J. Magn. Magn. Mater. 240, 273 (2002).

[11] L. Smardz, K. Le Dang, H. Niedoba, K. Chrzumnicka, J. Magn. Magn. Mater. 140-144, 569 (1995).

[12] L. Smardz, Sol. State Com. 112, 693 (1999).

[13] L. Smardz, J. Alloys Comp. 395, 17 (2005).

[14] L. Smardz, K. Smardz, H. Niedoba, J. Magn. Magn. Mater. 220, 175 (2000).

[15] J. Skoryna, A. Marczyńska, M. Lewandowski, L. Smardz, J. Alloys Comp. 645, 280 (2015). 
[16] J. Skoryna, M. Wachowiak, A. Marczyńska, A. Rogowska, Ł. Majchrzycki, W. Koczorowski, R. Czajka, L. Smardz, Surf. Coat. Techn. 303, 119 (2016).

[17] J. Skoryna, S. Pacanowski, A. Marczyńska, M. Werwiński, Ł. Majchrzycki, R. Czajka, L. Smardz, Surf. Coat. Techn. 303, 125 (2016).

[18] L. Smardz, M. Nowak, M. Jurczyk, Int. J. of Hydrogen Energy 37, 3659 (2012).

[19] L. Smardz, M. Jurczyk, K. Smardz, M. Nowak, M. Makowiecka, I. Okońska, Renewable Energy 33, 201 (2008).
[20] K. Smardz, L. Smardz, I. Okonska, M. Nowak, M. Jurczyk, International Journal of Hydrogen Energy 33, 387 (2008).

[21] A. Marczyńska, J. Skoryna, B. Szymański, L. Smardz, Acta Phys.Pol. A 127, 552 (2015).

[22] A. Marczyńska, J. Skoryna, L. Smardz, Acta Phys. Pol. A 126, 1315 (2014).

[23] D. Briggs, M.P. Seah, Practical Surface Analysis, John Wiley \& Sons, New York 1990. 